(Aus dem medicin.-chem. Laboratorium, der k. k. deutschen Universität in Prag.)

\title{
Ueber das von Freund und Töpfer angegebene Verfahren zur quantitativen Be- stimmung des Harnstoffs im Harn.
}

\section{Von \\ Hugo Pollak.}

Freund und Töpfer ${ }^{1}$ ) sind der Ansicht, dass die derzeit üblichen Methoden zur Bestimmung des Harnstoffs im Harn weder an Schnelligkeit noch an Verlässlichkeit der Ausführung den nöthigen Anforderungen genügen, und haben deshalb ein Verfahren in Vorschlag gebracht, welches nach ihrer Meinung von diesen Mängeln frei ist. Die Bestimmung ist in folgender Weise auszuführen.

Man soll 5 cem Harn unter Zusatz eines gleichen Volumens 95\% \%gen Alkohols auf dem Wasserbade zur Trockne abdampfen und den Rückstand im Moment des Trockenwerdens mit wenigen Tropfen Alkohol versetzen; der Rückstand wird dann nicht syrupös, sondern nimmt eine Beschaffenheit an, bei welcher er sich leicht mit Alkohol zerreiben und ausziehen lässt. Diesen Rückstand extrahirt man wiederholt mit absolutem Alkohol und filtrirt den Auszug in ein Kjeldahlkölbchen, aus welchem man den Alkohol bis auf Spuren verdunstet. Der dabei gewonnene Rückstand wird darauf mit ungefähr $70 \mathrm{ccm}$ gesättigter ätherischer Oxalsäurelösung übergossen und, wenn sich der Niederschlag abgesetzt hat, die Lösung durch ein Filter vorsichtig vom Niederschlag abgegossen. In derselben Weise wäscht man den Niederschlag in mehreren Portionen noch mit $60-80 \mathrm{cem}$ Aether. Nachdem der Aether vom Filter verdunstet ist, wird der auf ihm befindliche Niederschlag mit Wasser in das Kölbchen gewaschen und in der so entstandenen Lösung des gesammten Niederschlags die Oxalsäure mit Hülfe von Phenolphtaleïn titrirt. Darnach führt man mit dem Kolbeninhalt die Stickstoffbestimmung nach Kjeldahl aus. Aus diesen zwei Befunden, nämlich der Menge der gefundenen Oxalsäure und der des Stickstoffs, wird der Harnstoff berechnet. Ein Gehalt des Harns an Ei-

1) E. Freund und G. Töpfer, Wiener klinische Rundschau 13. Jahrgang Nr. 23 S. 371.1899. 
weiss oder Zucker habe keinen Einfluss auf die Genauigkeit der Methode.

Die Brauchbarkeit des Verfahrens haben Freund und Töpfer in der Weise geprüft, dass sie in verschiedenen Harnen den Harnstoff nach ibrem Verfahren bestimmten, sie dann mit einer bekannten Menge Harnstoff versetzten und die Bestimmung nochmals ausführten. In vier Fällen wurde dem analysirten Harn jedes Mal 3,94\% Harnstoff hinzugefügt und durch Titriren der Oxalsäure 3,87-3,90\%, durch die Stickstoffbestimmung 3,89-3,92\% Harnstoff wiedergefunden.

Demnach scheint das Resultat ein ausserordentlich günstiges zu sein. Die Art der Beweisführung kann aber nicht ohne Weiteres als zuverlässig angesehen werden; denn wenn die Bestimmung des Harnstoffs im nativen Harn mit einem (positiven oder negativen) Fehler behaftet wäre, so braucht dieser bei der Bestimmung der zugesetzten Menge Harnstoff doch nicht oder nicht in dem Maasse wie anfangs zum Ausdrucke zu kommen.

Gegen die Verlässlichkeit des Verfahrens bestehen auch insofern Bedenken, als dasselbe einen Verlust an Harnstoff nicht ausschliesst. Dieser Verlust kann eintreten durch Zersetzung des Harnstoffs beim Eindampfen, und ferner dann, wenn der oxalsaure Harnstoff in der Waschflüssigkeit nicht völlig unlöslich ist.

Es ist bekannt, dass sich der Harnstoff bereits in wässriger Lösung beim Erwärmien auf $100^{\circ}$ zersetzt; im Harn scheint diese Zersetzung durch die Gegenwart des zweifachsauren Phosphats unter Verlust von Ammoniak befördert zu werden. Es ist daher nicht einzusehen, wie nach F reu nd und $\mathrm{T}$ ö p fe r nach Vergrösserung des Harnvolumens durch Alkohol der Harn ganz ohne Gefahr der Zerstörung von Harnstoff eingedampft werden könne. Geht nun aber durch Zersetzung des Harnstoffs oder durch die theilweise Löslichkeit seines Oxalats Harnstoff verloren, so ist begreiflicher Weise mit der Uebereinstimmung der Oxalsäure und der Stickstoffbestimmung die Richtigkeit der Analyse nicht erwiesen. Das ist die schwache Seite des neuen Verfahrens.

Um ein selbstständiges Urtheil über den Werth des neuen Verfahrens zu gewinnen, erschien eine Wiederholung desselben geboten. Diese Untersuchung wurde auf Veranlassung von Prof. Huppert und unter seiner Leitung vorgenommen.

Zuerst habe ich mit denselben Harnen Harnstoffbestimmungen vergleichsweise nach Freund und Töpfer, sowie nach dem Verfahren von Schöndorff, dem jetzt am besten verbürgten, ausgeführt. 
und das Verfahren dann auf die Bestimmung von Harnstoff in reinen Harnstofflösungen ausgedehnt.

Die beiden Arten der Harnstoff bestimmung im Harn unterscheiden sich dadurch, dass nach Freund und Töpfer der Harnstoff als solcher, und zwar dieser allein, abgeschieden und bestimmt werden soll, während Schöndorff nach unserer jetzigen Kenntniss der Harnbestandtheile alle anderen stickstoffhaltigen Substanzen ausschliesst, bis auf das Allantoin und das Kreatin. Nach einer von Pod u schka ${ }^{1}$ ) unter der Leitung von Prof. Pohl ausgeführten Untersuchung kommt aber Allantoin im normalen Menschenharn, wenn überhaupt, höchstens in Spuren vor, und Kreatin ist in saurem Harne nicht oder wenigstens nicht in grosser Menge zu gewärtigen. Im schlimmsten Falle könnte nach dem Verfahren von Schöndorff etwas zu viel Harnstoff gefunden werden. Wie sich aber im Laufe dieser Untersuchung gezeigt hat, bängt die Entscheidung der Frage nach dem Werth des neuen Verfahrens nicht hiervon allein ab.

An dem Verfahren von Schöndorff haben wir einige unwesentliche A enderungen vorgenommen. $\mathrm{Cm}$ einen allzu grossen Ueberschuss an Phosphorwolframsäure zu vermeiden, wurde die zur Fällung erforderliche Menge in der Weise ermittelt, dass nach dem Versetzen von $10 \mathrm{ccm}$ Harn mit dem gleichen Volumen des (salzsäurebaltigen) Reagens die Menge des Reagens immer um nur $5 \mathrm{ccm}$ gesteigert wurde. Ferner wurde der abgemessene Theil des Filtrats, welches mit Kalkhydrat übersättigt worden war, so weit mit Wasser aufgefüllt, dass $5 \mathrm{ccm}$ je $1 \mathrm{ccm}$ Harn enthielten. Davon wurden $25 \mathrm{ccm}$ für die Harnstoffbestimmung verwendet. Es wird so zuletzt das unbequeme and zu Irrungen Anlass gebende Rechnen mit Brüchen vermieden. Endlich haben wir die $25 \mathrm{ccm}$ der Flüssigkeit, in welchen der Harnstoff bestimmt werden sollte, nach Zusatz der Phosphorsäure nicht sogleich in den Trockenschrank gebracht, sondern aus dem schräg gelagerten Kölbchen so lange Wasser weggekocht, bis sich die Flüssigkeit zu bräunen anfing. Bis dahin erreicht die Temperatur der Flüssigkeit nicht $150^{\circ} \mathrm{C}$. Die Kölbchen kamen darauf noch 3-4. Stunden in den Trockenschrank.

Es sei ausdrücklich betont, dass auch die Filtrate vom PhosphorwolframsäureNiederschlag wasserklar waren, also kein phosphorwolframsaures Arnmon enthielten.

In allen Versuchen wurde die Oxalsäure so wie bei Kjeldahl die Schwefelsäure mit 0,1 Normal-Natron titrirt; bei der Oxalsäure entspricht $0,1 \mathrm{ccm}$ derselben $0,6 \mathrm{mg}$ Harnstoff, bei Kjeldahl $0,1 \mathrm{cem}$ $0,3 \mathrm{mg}$ Harnstoff. Die folgenden zwei Tabellen geben an, wieviel Milligramm Harnstoff in je $5 \mathrm{ccm}$ Harn gefunden wurden. Unter Differenz ist die Abweichung der Resultate nach Schöndorff von den beiderlei Resultaten nach Freund und Töpfer zu verstehen. Die Ergebnisse der einzelnen Titrationen werden angeführt, weil sie ein Maass abgeben für die Genauigkeit der Bestimmung.

1) R. Poduschka, Archiv für experim. Pathologie Bd. 44 S. 62. 1900. 


\section{Versuche mit Harn.}

A. Normale Harne. Tabelle I.

\begin{tabular}{|c|c|c|c|c|c|c|c|c|c|}
\hline \multirow{4}{*}{ Nr. } & \multirow{2}{*}{\multicolumn{2}{|c|}{$\frac{\text { Schöndorff }}{\text { Harnstoff }}$}} & \multicolumn{6}{|c|}{ Freund und Töpfer } & \multirow{4}{*}{ Bemerkungen } \\
\hline & & & \multicolumn{6}{|c|}{ Harnstoff } & \\
\hline & & \multirow{2}{*}{ Mittel } & \multicolumn{3}{|c|}{ aus der Oxalsäure } & \multicolumn{3}{|c|}{ nach Kj eldah I } & \\
\hline & & & & Mittel & |Differenzi| & & Mittel & Differenz & \\
\hline \multirow[b]{2}{*}{1} & \multirow[t]{2}{*}{$\begin{array}{l}74,85 \\
74,55\end{array}$} & \multirow[t]{2}{*}{74,70} & $\begin{array}{l}73,8 \\
70,2\end{array}$ & 72,0 & $-2,7$ & $\begin{array}{l}69,9 \\
64,5\end{array}$ & 67,2 & $-7,5$ & \\
\hline & & & $\begin{array}{l}77,4 \\
75,6\end{array}$ & 76,5 & $+1,8$ & $\begin{array}{l}73,8 \\
75,9\end{array}$ & 74,85 & $+0,15$ & \\
\hline 2 & $\begin{array}{l}78,9 \\
78,6\end{array}$ & 78,75 & $\begin{array}{l}63,0 \\
79,8\end{array}$ & ? & $?$ & $\begin{array}{l}82,5 \\
80,7\end{array}$ & 81,6 & $+2,85$ & \\
\hline 3\{ & $\begin{array}{l}78,3 \\
76,5\end{array}$ & 77,4 & $\begin{array}{l}94,2 \\
95,4\end{array}$ & 94,8 & $+17,4$ & $\begin{array}{l}72,6 \\
72,3\end{array}$ & 72,45 & $-4,95$ & \\
\hline 4\{ & $\begin{array}{l}61,5 \\
62,7\end{array}$ & 62,1 & $\begin{array}{l}76,8 \\
72,0\end{array}$ & 74,4 & $+12,3$ & $\begin{array}{l}53,7 \\
50,7\end{array}$ & 52,2 & $-9,9$ & \\
\hline 5\{ & 83,5 & & $\begin{array}{l}96,6 \\
94,2\end{array}$ & 95,4 & $+11,9$ & $\begin{array}{l}75,0 \\
72,6\end{array}$ & 73,8 & $-9,7$ & \\
\hline 6 & $\begin{array}{l}72,3 \\
71,7\end{array}$ & 144,0 & $\begin{array}{l}145,8 \\
137,4 \\
144,6 \\
137,4 \\
136,2 \\
154,2\end{array}$ & 142,6 & $-1,4$ & $\begin{array}{l}132,0 \\
117,0 \\
-\overline{144,0} \\
125,55 \\
133,2\end{array}$ & 130,35 & $-13,65$ & $\left\{\begin{array}{l}\text { ZuSchóndorff auf } \\
\text { das Doppelte ver- } \\
\text { dünnt }\end{array}\right.$ \\
\hline & $\begin{array}{l}71,2 \\
71,1\end{array}$ & $\begin{array}{l}142,4 \\
142,2 \\
\end{array}$ & $\begin{array}{l}96,0 \\
84,0\end{array}$ & ? & $?$ & $\begin{array}{l}66,6 \\
66,9\end{array}$ & 133,5 & $-9,6$ & $\left\{\begin{array}{l}\text { Zu Freund u. Top- } \\
\text { fer auf das Dop- } \\
\text { pelte verdünit }\end{array}\right.$ \\
\hline & & 143,3 & $\begin{array}{l}125,4 \\
132,6\end{array}$ & 129,0 & $-14,3$ & $\begin{array}{l}122,7 \\
117,15\end{array}$ & 119,93 & $-23,37$ & 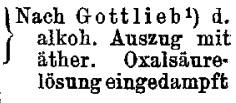 \\
\hline 7 & $\begin{array}{l}101,1 \\
100,35\end{array}$ & 100,72 & 134,7 & 185,45 & $+34,73$ & $\begin{array}{l}104,7 \\
105,3\end{array}$ & 105,0 & $+4,28$ & \\
\hline 1 & $\begin{array}{l}52,95 \\
52,20\end{array}$ & 105,15 & 136,2 & & $+30,3$ & & & $-0,15$ & $\left\{\begin{array}{l}\text { Das zweite Par zu } \\
\text { Schöndorffa.d. } \\
\text { Doppeite rexdünnt }\end{array}\right.$ \\
\hline 8 & $\begin{array}{c}104,55 \\
102,90 \\
52,2 \\
51,6\end{array}$ & $\begin{array}{l}103,73 \\
103,80\end{array}$ & $\begin{array}{r}121,2 \\
112,2 \\
130,8 \\
79,8\end{array}$ & 111,0 & $+7,2\}$ & \begin{tabular}{|c}
101,4 \\
96,15 \\
98,1 \\
93,45
\end{tabular} & 97,28 & $-6,4$ & $\begin{array}{l}\text { Bei dem zweiten Paar } \\
\text { wie bei } 7\end{array}$ \\
\hline 9 & $\begin{array}{l}54,9 \\
54,0\end{array}$ & 108,9 & $\begin{array}{l}115,8 \\
112,2\end{array}$ & 114,0 & $+5,1$ & $\begin{array}{l}103,2 \\
102,3\end{array}$ & 102,75 & $-6,15$ & $\left\{\begin{array}{l}\text { ZuSchōnd orff auf } \\
\text { das Doppelte rer- } \\
\text { dünnt }\end{array}\right.$ \\
\hline
\end{tabular}

1) R. Gottlieb, Archiv für experim. Pathologie Bd. 42 S. 242.

E. P flŭ g er, Arehiv für Physiologie. Bd. 88. 
B. Pathologische Harne. Tabelle II.

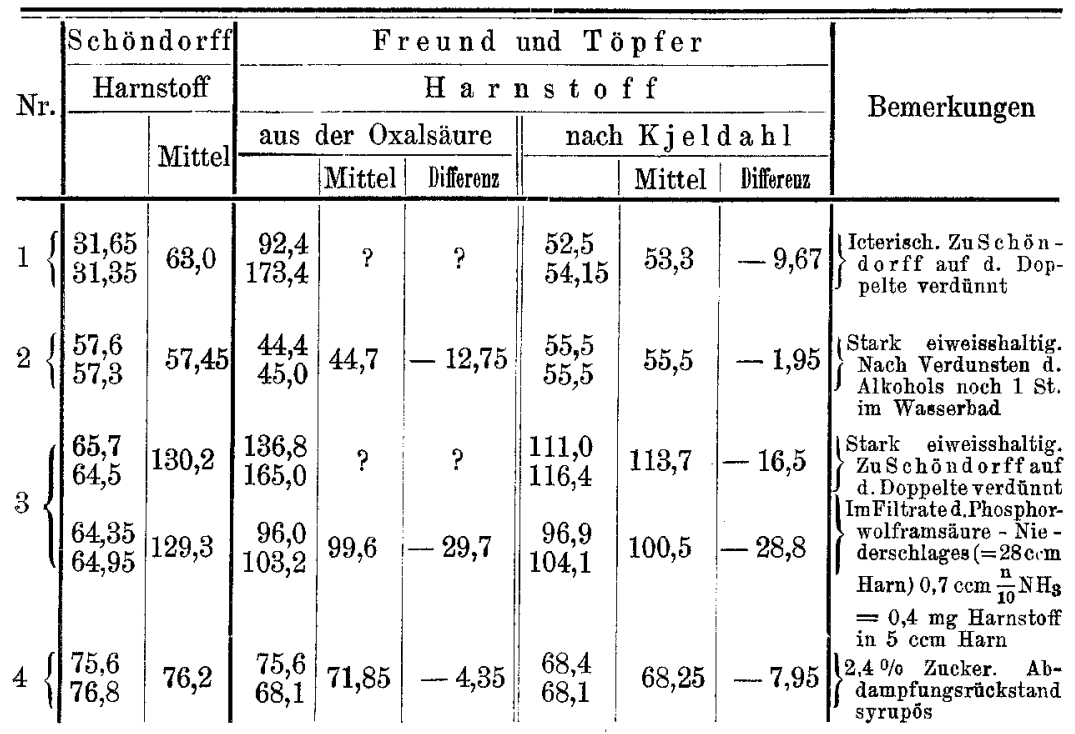

Vergleicht man zunächst die Harnstoffzahlen, wie sie bei dem Verfahren von Freund und Töpfer durch die Ammoniakbestimmung erhalten wurden, mit den nach Scbönd orff gefundenen, so stellt sich nur bei drei normalen Harnen (Nr. 1, 2 und 7 der Tabelle I) und bei einem eiweisshaltigen (Nr. 2 der Tabelle II) eine genügende Uebereinstimmung heraus. In den übrigen Fällen wurde nach Freund und Töpfer immer weniger Harnstoff bestimmt als nach Schöndorff; bei den normalen Harnen schwankt die Differenz, wenn man von dem nach Gottlieb untersuchten Harn absieht, zwischen 4,95 und $13,65 \mathrm{mg}$ und bei den pathologischen zwischen 7,9 und $28,8 \mathrm{mg}$. Diese Abweichungen können nicht aus einer mangelhaften Ausführung der Analyse erk]ärt werden, denn die starke Abweichung, welche sich bei dem normalen Harn Nr. 6 herausstellte, war Anlass dazu, dass die Analyse sowohl nach Schöndorff als auch nach Freund und Töpfer mehrmals wiederholt wurde; aber das Resultat wurde nicht besser. Ebenso verhielt es sich bei dem Eiweissharn Nr. 3. In diesem Falle wurde das Filtrat vom Phosphorwolframsäureniederschlag, obwohl es ganz klar war, einer Ammoniakbestimmung durch Destillation mit Kalkmilch im Vacuum unterzogen; die dabei gefundene, die Grenze der Versuchsfehler kaum überschreitende Menge Ammoniak hätte für die verwendeten $5 \mathrm{ccm}$ Harn nur $0,4 \mathrm{mg}$ Harnstoff ausgemacht. 
Diese Abweichungen zwischen den beiderlei Harnstoffbestimmungen würden aus einem Verlust bei dem Verfabren von Freund und Töpfer zu erklären sein, wenn man das Verfahren von Schöndorff als zuverlässig ansieht. Bei dem Eiweissharn Nr. 8 könnte man daran denken, dass das Eiweiss der Extraction des Harnstoffes hinderlich ist, wenn nicht gerade der andere Eiweissharn (Nr. 2) ein sebr günstiges Resultat ergeben hätte. Für die übrigen Fälle wäre die Quelle des Verlustes noch aufzusuchen.

Die Harnstoffbestimmungen aus der Titrirung der Oxalsäure nach Freund und Töpfer haben kein besseres Resultat geliefert als die Stickstoffbestimmungen. Auch hier sind nur wenige (Nr. 1 und Nr. 6 der Tabelle I und allenfalls Nr. 4 der Tabelle II) im Vergleich zur Harnstoffbestimmung nach Schöndorff befriedigend ausgefallen. Die meisten anderen weisen einen Mehrbetrag an Harnstoff (bis 34,7 mg) auf und diejenigen drei pathologischen, in welchen die einzelnen Oxalsäurebestimmungen nahe übereinstimmen, einen Feblbetrag (bis 29,7 mg Harnstoff). Von dem Ueberschuss kann man vermuthen, dass er dadurch zu Stande gekommen ist, dass sich aus dem Oxalsäureniederschlag bei Befolgung der von Freund und Töpfer gegebenen Vorschrift die freie Oxalsäure nicht vollständig auswaschen lässt; für die Erklärung des Verlustes von Oxalsäure gibt der Umstand einen Anhalt, dass mit einem auffallend grossen Verlust von Stickstoff auch der Verlust an Oxalsäure ein erheblicher war (Nr. 6 der Tabelle I und Nr. 3 der Tabelle II). Bei Wiederholung der Bestimmung änderte sich auch hier das Resultat nicht wesentlich, wie aus den Analysen der normalen Harne 1,6 und $7 \mathrm{zu}$ ersehen ist.

Endlich ist noch zu bemerken, dass sich zwischen der Stickstoffbestimmung bei Freund und Töpfer und der Bestimmung der Oxalsäure, wenn man von den oben erwähnten zwei Fällen absieht, keinerlei Beziehungen herausstellen. Das ist auch begreiflich, wenn man annimmt, dass einerseits die freie Oxalsäure nicht vollständig entfernt wird, andererseits ein Verlust an Harnstoff stattfindet.

An den Beobachtungen am Harn allein lässt sich die Richtigkeit dieser Annahme freilich nicht prüfen. Bessere Auskunft war dagegen von Versuchen mit Lösungen von reinem Harnstoff zu erwarten. 


\section{Versuche mit Harnstoff.}

Der verwendete Harnstoff war in der Kälte aus Aetheralkohol umkrystallisirt und im Vacuum über Schwefelsäure getrocknet worden. Aus demselben wurde eine Lösung mit $2 \mathrm{~g}$ in $100 \mathrm{~cm}$ Wasser hergestellt. Die Stickstoffbestimmung nach Kjeldahl ergab in $5 \mathrm{ccm}$ der Lösung (=100 mg) 99,9 und 100,8, im Mittel 100,35 mg Harnstoff. Der Harnstoff war also als rein zu betrachten.

Zuerst wurden von dem Harnstoff selbst abgewogene Mengen im Kjeldahlkolben in dem möglichst geringsten Volumen absoluten Alkohols gelöst, dann mit ätherischer Oxalsäurelösung gefällt und der Niederschlag nach Freund und Töpfer weiter behandelt. Diese Form des Versuches wurde gewählt, um Verluste an Harnstoff zu vermeiden, welche durch das Eindampfen der Lösung und beim Uebertragen des alkoholischen Auszugs in den Kjeldahlkolben eintreten konnten.

Tabelle III.

\begin{tabular}{c|c|c}
\hline \multirow{2}{*}{ Angewandt } & \multicolumn{2}{|c}{ G e f u n d e $\mathrm{n}$} \\
\cline { 2 - 3 } & aus der Oxalsäure & nach Kjeld ahl \\
\hline $96,3 \mathrm{mg}$ & $99,3 \mathrm{mg}$ & $90,3 \mathrm{mg}$ \\
$152,2 \mathrm{mg}$ & $160,2 \mathrm{mg}$ & $144,3 \mathrm{mg}$
\end{tabular}

Aus der Stickstoffbestimmung geht hervor, dass bei der Behandlung der alkoholischen Harnstofflösung mit der ätherischen Oxalsäurelösung in der That ein nicht unerheblicher Verlust an Harnstoff stattfindet. Die Titrirung der Oxalsäure ergab dagegen einen Ueberschuss, und dieses Resultat ist sicher nur daraus zu erklären, dass der Harnstoffniederschlag von der Oxalsäure nicht völlig freigewaschen worden war.

Mit der oben erwähnten Harnstofflösung wurden ferner vollständige Analysen nach Freund und Töpfer ausgefuhrt; sie sollten lehren, welchen Einfluss das Eindampfen der Harnstofflösung mit Alkohol auf den Ausfall der Bestimmung haben könne. 
Tabelle IV.

\begin{tabular}{|c|c|c|c|c|}
\hline \multirow{3}{*}{ Angewandt } & \multicolumn{4}{|c|}{$G$ e f u $n d e n$} \\
\hline & \multicolumn{2}{|c|}{ aus der Oxalsäure in $\mathrm{mg}$} & \multicolumn{2}{|c|}{ nach $\mathrm{Kjeldahl}$ in $\mathrm{mg}$} \\
\hline & & Mittel & & Mittel \\
\hline $100 \mathrm{mg}$ Harnstoff & $\begin{array}{l}92,4 \\
93,6\end{array}$ & 93,0 & $\begin{array}{l}91,95 \\
91,50\end{array}$ & 91,72 \\
\hline $100 \mathrm{mg}$ Harnstoff & $\begin{array}{r}99,3 \\
102,6\end{array}$ & 100,95 & $\begin{array}{l}91,05 \\
91,95\end{array}$ & 91,50 \\
\hline $200 \mathrm{mg}$ Harnstoff & $\begin{array}{l}191,4 \\
195,6\end{array}$ & 193,5 & $\begin{array}{l}188,1 \\
188,7\end{array}$ & 188,40 \\
\hline
\end{tabular}

Auch hier ergab die Stickstoff bestimmung einen Verlust an Harnstoff, der noch etwas grösser war, als wenn das Eindampfen unterlassen wurde. In Uebereinstimmung steht damit, dass gleichfalls weniger Oxalsäure nachgewiesen wurde als in den in Tabelle III angeführten Versuchen mit festem Harnstoff.

Wurde der Harnstofflösung vor dem Eindampfen ungefähr so viel zweifachsaures Phosphat hinzugefügt, als im Verhältnisse zum Harnstoff der Harn enthält, so war, wie Tabelle V zeigt, der Verlust an Stickstoff sowohl wie an Oxalsäure noch etwas grösser, als wenn der Zusatz an Phosphat unterblieb.

Tabelle V.

\begin{tabular}{|c|c|c|c|c|}
\hline \multirow{3}{*}{ Angewandt } & \multicolumn{4}{|c|}{$\mathrm{G}$ e $\mathbf{f} \boldsymbol{u} \mathbf{n} \mathbf{d}$ e $\mathbf{n}$} \\
\hline & \multicolumn{2}{|c|}{ aus der Oxalsäure in $\mathrm{mg}$} & \multicolumn{2}{|c|}{ nach $\mathrm{Kjeldahl}$ in $\mathrm{mg}$} \\
\hline & & Mittel & & Mittel \\
\hline \multirow{2}{*}{$\begin{array}{l}100 \mathrm{mg} \text { Harnstoff mit } \\
1,4 \mathrm{ccm} \frac{\mathrm{n}}{16} \mathrm{KH}_{2} \mathrm{PO}_{4}\end{array}$} & $\begin{array}{l}90,6 \\
93,6\end{array}$ & 92,1 & $\begin{array}{l}90,6 \\
87,0\end{array}$ & 88,80 \\
\hline & $\begin{array}{l}92,1 \\
93,6\end{array}$ & 92,85 & $\begin{array}{l}91,8 \\
91,2\end{array}$ & 91,50 \\
\hline $\begin{array}{l}100 \mathrm{mg} \text { Harnstoff mit } \\
2,7 \mathrm{ccm} \frac{\mathrm{n}}{16} \mathrm{KH}_{2} \mathrm{PO}_{4}\end{array}$ & $\begin{array}{l}71,4 \\
8:, 4\end{array}$ & $?$ & $\begin{array}{l}90,9 \\
89,7\end{array}$ & 90,30 \\
\hline $\begin{array}{l}100 \mathrm{mg} \text { Harnstoff mit } \\
2,8 \mathrm{ccm} \frac{\mathrm{n}}{16} \mathrm{KH}_{2} \mathrm{PO}_{4}\end{array}$ & $\begin{array}{l}89,1 \\
87,9\end{array}$ & 88,5 & $\begin{array}{l}86,7 \\
87,0\end{array}$ & 86,85 \\
\hline
\end{tabular}

Nach dem Ausfall der Versuche mit reinem Harnstoff wird man also annehmen dürfen, dass der Unterschied zwischen der Stickstoff- 
bestimmung nach dem Verfabren von Schöndorff und dem von Freund und Töpfer seinen Grund hat in einem Verlust an Harnstoff bei der Methode von Freund und Tö pfer. Dieser Verlust wird bedingt durch eine theilweise Zersetzung des Harnstoffs beim Eindampfen und durch die nicht völlige Unlöslichkeit des oxalsauren Harnstoffs in Aether oder wenigstens in dem anfangs vorhandenen schwach alkoholhaltigen Aether.

Die Bestimmung des Harnstoffs nach der Menge der titrirten Oxalsäure ist mit noch viel grösseren Fehlern behaftet als die Bestimmung des Harnstoffs nach dem Stickstoffgehalt des Oxalsäureniederschlags. Es wird zu viel Oxalsäure gefunden, wenn der Niederschlag ungenügend ausgewaschen ist, und zu wenig bei vollständigem Auswaschen, wegen der Löslichkeit des oxalsauren Harnstoffs. Zuweilen ist die Titrirung unsicher, weil die Lösung des Niederschlages gefärbt ist.

In der Vorschrift zur Ausführung ihrer Analyse geben Freund und Töpfer ausdrücklich an, man solle nach der Titrirung der Oxalsäure noch die Stickstoff bestimmung ausführen; es würde somit die eine Bestimmung zur Controle der anderen dienen. Wenn aber auch die Stickstoff bestimmung vorgenommen werden soll, so ist nicht einzusehen, wie dann die von Freund und Töpfer bei den derzeit üblichen Methoden vermisste Schnelligkeit der Ausführung erreicht werden soll. Man braucht zu dieser Bestimmung ebenso viel Zeit wie zu der Harnstoffbestimmung nach Schöndorff, und dazu kommt noch, dass das Verfahren von Freund und Töpfer viel grössere Ansprüche an die Fertigkeit des Analytikers stellt als das Verfahren von Schöndorff. Wie die von mir mitgetheilten Belege (namentlich Tabelle I6) darthun, ergibt dieses bei wiederholten Analysen völlig befriedigende Uebereinstimmung.

Einen Vortheil böte das Verfahren von Freund und Töpfer dar, wenn man sich auf die Titrirung der Oxalsäure allein beschränken könnte. Die von mir gemachten Erfahrungen sind dieser Annahme nicht günstig. Ich gebe gern $\mathrm{zu}$, dass eine bessere Uebereinstimmung zwischen der Stickstoffbestimmung und der Titrirung der Oxalsäure erreicht werden kann, als es in meinen Beobachtungen der Fall ist, nämlich dann, wenn man aus dem Niederschlag die freie Oxalsäure völlig wegwäscht; aber dann bleibt doch der wesentliche Fehler des Verfahrens, der Verlust an oxalsaurem Harnstoff, noch bestehen, und das Verfahren selbst wird dadureh nicht gebessert. 